\title{
Nonlinear bandgap opening behavior of BN co-doped graphene
}

Bo-Yao Wang ${ }^{\mathrm{a}}$, Hsiaotsu Wang ${ }^{\mathrm{b}}$, Ling-Yen Chen ${ }^{\mathrm{c}}$, Hung-Chung Hsueh ${ }^{\mathrm{c},}$. $\mathrm{Xin} \mathrm{Li}^{\mathrm{d}, \mathrm{e}}$, Jinghua Guo ${ }^{\mathrm{d}}$, Yi Luo ${ }^{\mathrm{e}}$, Jau-Wern Chiou ${ }^{\mathrm{f}}$, Wei-Hua Wang ${ }^{\mathrm{g}}$, Po-Hsiang Wang ${ }^{\mathrm{g}}$, Kuei-Hsien Chen ${ }^{\mathrm{g}}$, Yen-Chih Chen ${ }^{\mathrm{h}}$, Li-Chyong Chen ${ }^{\mathrm{h}}$, Chia-Hao Chen ${ }^{\mathrm{i}}$, Jian Wang, Way-Faung Pong ${ }^{\mathrm{c}, *}$

${ }^{a}$ Department of Physics, National Changhua University of Education, Changhua 500, Taiwan

${ }^{\mathrm{b}}$ Department of Physics, National Tsinghua University, Hsinchu 300, Taiwan

${ }^{\mathrm{c}}$ Department of Physics, Tamkang University, Tamsui 251, Taiwan

d Advanced Light Source, Lawrence Berkeley National Laboratory, Berkeley, CA 94720, USA

${ }^{\mathrm{e}}$ Department of Theoretical Chemistry and Biology, School of Biotechnology, KTH Royal Institute of Technology, S-10691, Stockholm, Sweden

${ }^{\mathrm{f}}$ Department of Applied Physics, National University of Kaohsiung, Kaohsiung 811, Taiwan

${ }^{\mathrm{g}}$ Institute of Atomic and Molecular Sciences, Academia Sinica, Taipei 106, Taiwan

${ }^{\mathrm{h}}$ Center for Condensed Matter Sciences, National Taiwan University, Taipei 106, Taiwan

${ }^{\mathrm{i}}$ National Synchrotron Radiation Research Center, Hsinchu 300, Taiwan

${ }^{\mathrm{j}}$ Canadian Light Source Inc., University of Saskatchewan, Saskatoon S7N 2V3, Canada

We have demonstrated a nonlinear behavior for the bandgap opening of doped graphene by controlling the concentration of $\mathrm{B}$ and $\mathrm{N}$ co-dopants. X-ray absorption and emission spectra reveal that the bandgap increases from 0 to $0.6 \mathrm{eV}$ as the concentration of $\mathrm{BN}$ dopants is increased from 0 to $6 \%$, while the bandgap closes when the doping concentration becomes $56 \%$. This nonlinear behavior of bandgap opening of the $\mathrm{BN}$-doped graphene depending on the $\mathrm{BN}$ concentrations is consistent with the valence-band photoemission spectroscopic measurements. The spatially resolved $\mathrm{B}, \mathrm{N}$ and $\mathrm{C} K$-edge scanning transmission x-ray microscopy and their $\mathrm{x}$-ray absorption near-edge structure spectra all support the scenario of the development of 
$h$-BN-like domains at high concentrations of $\mathrm{BN}$. Ab initio calculation, by taking into account of the strong correlation between the bandgap and the geometry/concentration of the dopant, has been performed with various BN-dopant nano-domains embedded in the graphene monolayer to verify the unique bandgap behavior. Based on the experimental measurements and ab initio calculation, we propose the progressive formation of a phase-separated zigzag-edged $\mathrm{BN}$ domain from $\mathrm{BN}$ quantum dots with increasing $\mathrm{BN}$-dopant concentration to explain the extraordinary nonlinear behavior of bandgap opening of $\mathrm{BN}$-doped graphene sheets. This study reveals a new way to engineer the bandgap of low-dimensional systems.

Corresponding author.

E-mail addersses: hchsueh@mail.tku.edu.tw,wfpong@mail.tku.edu.tw 


\section{Introduction}

With the characteristics of a single atomic layer and high electron mobility, graphene is currently regarded as one of the most promising materials for use in the next generation of high-speed nanodevices [1,2]. However, pristine graphene has no bandgap at the Fermi level $\left(E_{\mathrm{f}}\right)$ at the $K$ point, which means that the highest occupied and lowest unoccupied states coincide at this $K$ point. The dispersion of the two corresponding energy bands is approximately linear near the $K$ point and differ in that respect from the parabolic dispersions close to the valence-band maximum $\left(E_{\mathrm{VBM}}\right)$ and the conduction-band minimum $\left(E_{\mathrm{CBM}}\right)$ of a semiconductor, which limits its semiconducting behavior and range of applications. To retrieve the bandgap, approaches such as size/shape and symmetry modification/breaking, hydrogenation, fluorination and functionalization have recently been developed [3-8]. However, the challenges in obtaining a reasonable bandgap while maintaining a compatible honeycomb structure have not yet been properly overcome. A promising solution involves engineering doping [9], which is a well-known method for modulating the electronic properties of graphene. Among various boron-nitrides, hexagonal boron-nitride $(h-\mathrm{BN})$ is an effective dopant for inducing a bandgap in graphene [10-20] because it has not only a wide bandgap (with an energy gap range from 3.6 to $7.1 \mathrm{eV}$ ) [21-25] but also has a similar honeycomb lattice to that of graphene. Doping with B 
and $\mathrm{N}$ atoms may induce a slight shift of $E_{\mathrm{f}}$, although these atoms have opposite charge-transfer effects ( $p$ - and $n$-type doping) $[13,19,20,26]$ thus providing the potential advantage of a low required gate voltage to give graphene an insulating property. UV-visible light [14] and preliminary bandgap measurements [15] have been performed to reveal the existence of a bandgap in the BN-co-doped graphene at particular doping concentrations. Despite many research works in this field [10-20,27], the mechanism of the opening of the bandgap and its correlation with the characters of $\mathrm{BN}$ dopant are not yet fully understood. Unsurprisingly, introducing $\mathrm{BN}$ dopant breaks the sublattice symmetry, and deviation from the gapless character of graphene is then expected [20]. According to previous theoretical studies, the bandgap of graphene is generated by either a local charge density and potential redistribution effect of small BN crystallites or a local quantum confinement effect associated with large $h$-BN domains $[3,13,14]$. However, these two physical inferences concerning the formation of the bandgap differ considerably. Ab initio calculations of the electronic structures of graphene doped with BN nanoribbons have revealed that the bandgap depends on the narrowest region of graphene, regardless of the concentration and shape of the BN regions [12]. Advanced GW and Bethe-Salpeter calculations have shown that the energy gap of carbon-boronnitride (C-BN) monolayers is determined only by the size of $\mathrm{C}$ domains, regardless of the overall composition of the system 
[28]. Quantum confinement in the finite-sized C domains of a mixed B-C-N system can also create a bandgap, though it exists within an inhomogeneous system using real-space pseudopotential density functional theory (DFT) [29]. Thus, the opening of the bandgap in $\mathrm{BN}$-doped graphene is expected to be strongly correlated with dopant concentrations and atomic arrangements [3,13,14,16-20], which can be controlled during its synthesis.

In this study, the bandgap opening of $\mathrm{BN}$-doped graphene depending on the doping concentrations is obtained by the electronics structure studies experimentally and theoretically. Surprisingly, the bandgap opening is found to be a nonlinear function of the total concentration of $\mathrm{BN}$ dopants. Initially, the bandgap increases from 0 to 0.6 $\mathrm{eV}$ as the concentration of $\mathrm{BN}$ dopants increases from 0 to $6 \%$; it then decreases and approaches zero when the $\mathrm{BN}$ concentration is increased further to $56 \%$. This nonlinear variation is strongly related to the electronic and bonding modifications or to the separation of phases by $\mathrm{BN}$ dopants in various doping regimes. The formation of randomly distributed small regions of $\mathrm{BN}$ dopants in the low-doping regime significantly changes the electronic states of the adjacent graphene. However, in the high-BN-doping regime, the formation of phase-separated $h$-BN-like and graphene domains is clearly observed in the BN-doped graphene samples herein. The proposed 
physical interpretation is supported by first-principles calculations based on the heterostructured $(\mathrm{BN})_{\mathrm{x}} \mathrm{C}_{\mathrm{y}}$ monolayers with specific local dopant ( $\mathrm{B}$ and $\mathrm{N}$ ) configurations in various concentrations.

\section{Experimental details}

$\mathrm{BN}$-doped graphene (1-2 layer) was grown on $\mathrm{Cu}$ foil by low-pressure chemical vapor deposition. Ammonia borane was introduced as the source of $\mathrm{B}$ and $\mathrm{N}$ atoms during the growth of graphene; its structural, electrical and optical properties were described in detail elsewhere [15]. The concentrations of the co-doped $\mathrm{B}$ and $\mathrm{N}$ atoms were controlled by varying the temperature of the precursor container and were determined by analyzing the intensity of core-level x-ray photoelectron spectroscopy (XPS) with an $\mathrm{Al} K_{\alpha}$ X-ray source $(1486.6 \mathrm{eV})$.

X-ray absorption near-edge structure (XANES) and x-ray emission spectroscopy (XES) measurements were carried out on beamlines 6.3.1.2 and 8.0.1 of the Advanced Light Source, Lawrence Berkeley National Laboratory. B, C and N $K$-edge XANES provided information on the conduction electronic (unoccupied) states and chemical bonding of the BN-doped graphene. The spectra were obtained by recording the surface-sensitive total electron yield as a function of x-ray photon energy. The valence electronic (occupied) states were measured by the $\mathrm{C}$ and $\mathrm{N} K_{\alpha}$ XES, with 
excitation energies of $320 \mathrm{eV}$ and $435 \mathrm{eV}$, respectively. The XANES and XES spectra were recorded at the angle of $45^{\circ}$ between the sample normal and the incident $\mathrm{x}$-ray. All XANES spectra were normalized to the incident beam intensity such that the areas under the spectra of the B, C and N XANES were fixed in the energy ranges 207-210 $\mathrm{eV}, 317-320 \mathrm{eV}$ and 427-430 eV, respectively. The energy scale of $\mathrm{B}, \mathrm{N}$ and $\mathrm{C}$ $K$-edge XANES spectra were also calibrated using $h$-BN and highly oriented pyrolytic graphite (HOPG) the as references. The XES spectra were calibrated using the elastic peaks at the same energies of the incident $\mathrm{x}$-ray. The energy resolutions of the $\mathrm{B}, \mathrm{C}$ and $\mathrm{N} K$-edge XANES and $K_{\alpha}$ XES were set to $0.2 \mathrm{eV}$ and $0.35 \mathrm{eV}$, respectively. The valence-band photoemission spectroscopic (VB-PES) spectra were obtained at BL-09A of the National Synchrotron Radiation Research Center in Hsinchu, Taiwan. The energy of the incident $\mathrm{x}$-ray was fixed at $150 \mathrm{eV}$, and the energy resolution was set to better than $0.1 \mathrm{eV}$. The VB-PES spectra were also calibrated using the $E_{\mathrm{f}}$ of pure gold. Scanning transmission x-ray microscopy (STXM) was conducted using the SM-beamline at the Canadian Light Source. A 25-nm-thick outermost-zone plate was used, providing a spatial resolution of $30 \mathrm{~nm}$ and a spectral resolution of better than $0.1 \mathrm{eV}$. Further details of the STXM-XANES measurement could be found elsewhere $[30,31]$. 


\section{Theoretical method}

The ground-state electronic structure and energies of the graphene-BN [heterostructural $(\mathrm{BN})_{x} \mathrm{C}_{\mathrm{y}}$ monolayers] with various local dopant configurations have been calculated using a full-potential projector augmented wave (PAW) scheme [32] in the Vienna ab initio simulation package (VASP) [33]. These calculations are based on DFT, which incorporates the exchange and correlation functional in a generalized gradient approximation (GGA) [34]. A cutoff energy of $500 \mathrm{eV}$ is utilized for the plane-wave basis set, and fine Monkhorst-Pack $k$-grid samplings [35] $(6 \times 6 \times 1 k$-grid for a 72-atom supercell) are performed on the Brillouin zone (BZ) integrals to ensure convergence. The underlying atomic positions in various $\mathrm{BN}_{x} \mathrm{C}_{1-x}$ monolayers are optimized from first-principles calculations until the total force on each atom is less than $0.001 \mathrm{eV} / \mathrm{A}$. The freestanding BN-doped graphene monolayer in low-dimensional systems is approximated as a single $(\mathrm{BN})_{\mathrm{x}} \mathrm{C}_{\mathrm{y}}$ layer surrounded by vacuum in a supercell. An inter-sheet separation of $15 \AA$ suffices to prevent unphysical interlayer interactions.

With respect to two components, i.e., 2D hexagonal $\mathrm{BN}$ domains and graphene of $(\mathrm{BN})_{x} \mathrm{C}_{y}$, the energy of formation per atom of $\mathrm{BN}_{(\mathrm{Q} e x)}\left(E_{f}^{(1)}\right)$ is defined as

$$
E_{f}^{(1)}=\frac{1}{N}\left(E_{(B N)_{x} C_{y}}^{Q D_{(\text {Hex }}}-\frac{x}{2 x+y} E_{B N}-\frac{y}{2 x+y} E_{C}\right)
$$


where $E_{(B N)_{x} C_{y}}^{Q D_{(H e x)}}$ is the calculated total energy of formation of $(\mathrm{BN})_{x} \mathrm{C}_{y}$ with the $\mathrm{BN}$ QD dopant. $E_{B N}$ and $E_{C}$ are the calculated total energies of formation of a $\mathrm{BN}$ monosheet and graphene sheet, respectively. $N$ is the number of atoms within the supercell. Similarly, the energy of formation per atom of phase-separated nanoribbon-type dopant $\left(E_{f}^{(2)}\right)$ is calculated as

$$
E_{f}^{(2)}=\frac{1}{N}\left(E_{(B N)_{x} C_{y}}^{p h-s e p}-\frac{x}{2 x+y} E_{B N}-\frac{x}{2 x+y} E_{C}\right)
$$

where $E_{(B N)_{x} C_{y}}^{p h-s e p}$ is the calculated total energy of $(\mathrm{BN})_{x} \mathrm{C}_{y}$ with zigzag-edged/armchair-edged nanoribbon-type dopants. Therefore, the relative structural stability can be determined by comparing the difference of corresponding formation energies per atom $\left(\Delta E_{f}\right)$,

$$
\Delta E_{f} \equiv E_{f}^{(2)}-E_{f}^{(1)}
$$

\section{Results and discussion}

Figure 1(a) presents the $\mathrm{C} K_{\alpha} \mathrm{XES}$ and $K$-edge XANES spectra of graphene that contains various concentrations of $\mathrm{BN}$ dopants. The references samples of pristine graphene and HOPG are included for comparison. The $\pi\left(\pi^{*}\right)$ feature at $277-284 \mathrm{eV}$ 
$(\sim 285.5 \mathrm{eV})$ is typically attributed to the graphitic-like $s p^{2}$ bond that is perpendicular to the plane, whereas the $\sigma\left(\sigma^{*}\right)$ feature at $270-277 \mathrm{eV}$ (above $292 \mathrm{eV}$ ) is attributed to the $\mathrm{C}-\mathrm{C}$ bond within the plane [36-40]. The general features of the $\mathrm{C} K_{\alpha} \mathrm{XES}$ and $K$-edge XANES spectra of the BN dopants, shown in Fig. 1(a), are consistent with the results in the literature. Feature $\boldsymbol{a}$ at $\sim 288.5 \mathrm{eV}$ in Fig. 1(a) is associated with C-B/C-N bonds with $\mathrm{BN}$ dopants [41] and/or the bonding of the oxygen-containing functional group and carbonboxyl/carbonyl group on the surface, which is associated with the surface and/or edge states $[37,38,42,43]$. Because the energy of feature $\boldsymbol{a}$ is far from $E_{\mathrm{CBM}}$ or $E_{\mathrm{f}}$, its effect on the bandgap opening is expected to be weak. Figure 1(b) magnifies the $\pi-\pi^{*}$ region of the spectra in Fig. 1(a). According to the results of $\mathrm{C}$ $K$-edge XANES [Figs. 1(a) and (b)], the intensity of $\sigma^{*}$ feature (above $292 \mathrm{eV}$ ) is gradually reduced when increases the BN concentration. Since $\sigma^{*}$ feature is known to be correlated with the $\mathrm{C}-\mathrm{C}$ bond within the plane of graphene, this variation indicates that the in-plane bonding configuration of $\mathrm{C}$ atoms is suppressed when the $\mathrm{B}$ and $\mathrm{N}$ atoms are increased. Therefore, this suggests the BN atoms are unlikely stacking only along out-of-plane of graphene but also embedded in the graphene matrix. Schematically, to obtain a precise value of the bandgap, the leading edges in both the C $K_{\alpha}$ XES and $K$-edge XANES spectra are extrapolated to the baselines to obtain $E_{\mathrm{VBM}}$ and $E_{\mathrm{CBM}}[37,38,44]$, respectively, as shown in Fig. 1(c), and to the bandgap of 
graphene with BN dopants. The two extrapolated lines in Fig. 1(b), for the 56\% $\mathrm{BN}$-doped graphene, pristine graphene and HOPG clearly intersect one another, which indicates that the $56 \% \mathrm{BN}$-doped graphene exhibits no bandgap that is similar to that of metallic graphene or HOPG [37,38]. Evidently, the bandgap opens to $\sim 0.2$ $\mathrm{eV}$ as the $\mathrm{BN}$ content increased to $2 \%$ and reaches a maximum $(\sim 0.6 \mathrm{eV})$ as the $\mathrm{BN}$ content increased to $6 \%$. This bandgap opening is confirmed by the results of the temperature dependence of resistivity made by Chang et al. [15], which indicates no bandgap of pristine graphene and the semiconductor behavior of low-BN-doped graphene. The measurements of the temperature dependence of resistivity of low-BN-doped graphene also reveal consistent findings (Fig. S1 in Supplementary Information). Surprisingly, the bandgap becomes smaller as the BN content increases to $26 \%(\sim 0.4 \mathrm{eV})$, and further the bandgap is closed in the $56 \% \mathrm{BN}$-doped graphene.

Figure 2 presents the $\mathrm{N} K_{\alpha}$ XES and $K$-edge XANES spectra of the BN-doped graphene with various $\mathrm{BN}$ concentrations and the reference $h$-BN. The features in the $\mathrm{N} K$-edge XANES spectra can be observed only when the doping BN concentration reaches 6\%; no clear XANES signature can be obtained from the $2 \% \mathrm{BN}$-doped graphene due to the low sensitivity of atomic absorption to the low concentration of $\mathrm{N}$ atoms. Furthermore, graphene that contains low BN concentrations (6\% and 26\%) 
yields $\mathrm{N} K_{\alpha}$ XES spectra of low statistics, so the bandgaps are not reliably to be determined. The intensities of the N $K_{\alpha}$ XES and $K$-edge XANES spectra of the $56 \%$ BN-doped graphene are much higher and are very close to the spectral shape of reference $h$-BN. This result confirms the presence of $h$-BN-like domains in the $56 \%$ BN-doped graphene, as discussed above. From the lines extrapolated to the baselines in both the XANES and XES spectra, as shown in Fig. 2, the bandgap of both $56 \%$ BN-doped graphene and $h$ - $\mathrm{BN}$ is estimated to be $\sim 4.4 \mathrm{eV}$, which is close to the experimental value in the literature [21-25]. It suggests that the nucleation of $\mathrm{BN}$ dopants at a low concentration forms N-B/N-C/B-C dimers and favors the formation of $h$-BN-like domains at a higher BN concentration of $56 \%$. The B $K$-edge XANES results show consistent findings (Fig. S2 in Supplementary Information). The development of $h$-BN-like domains is easier at high concentrations of $\mathrm{BN}$ because their formation energies are lower [13].

Figure 3 presents the spatial mappings of B, N and C for the $30 \% \mathrm{BN}$-doped graphene. Since the regions of the BN dopants in the graphene that was doped with a low concentration of $\mathrm{BN}$ are estimated to be smaller than the order of $\mathrm{nm}$, which is much smaller than the spatial resolution of STXM $(\sim 30 \mathrm{~nm})$, these dopants are difficult to detect. Therefore, this work focuses on the relatively highly doped $30 \% \mathrm{BN}$-doped 
graphene. With respect to the STXM mappings (left panels) and corresponding STXM-XANES (right panels) of the sample, the stack mappings are decomposed into blue, green, yellow and red maps (in the middle panels), which correspond to regions that are associated with different thicknesses and spectroscopic variations of the samples obtained by principle component analysis (PCA) for cluster analysis using the aXis2000 program [45-47]. The PCA spectrum of each of these three regions is average of spectra from all of the image pixels in that region. A more intense average spectrum generally indicates the sample thickness is higher, and all chemical species in that region contribute to the thickness. The STXM mapping images $\left(\mathrm{B}_{0}, \mathrm{~N}_{0}\right.$ and $\left.\mathrm{C}_{0}\right)$ of the blue region have almost zero optical density, corresponding to the zero intensity of the B, N and C $K$-edge STXM-XANES spectra, i.e. free of sample region. The STXM mapping images of the green, yellow and red regions (images $B_{1}-B_{3}, N_{1}-N_{3}$ and $\mathrm{C}_{1}-\mathrm{C}_{3}$ ) were images of regions that are distributed randomly in the samples. Notably, the intensity of STXM-XANES in the regions of $B_{3} / N_{3} / C_{3}$ (red curve) is nearly double that in the regions $\mathrm{B}_{1} / \mathrm{N}_{1} / \mathrm{C}_{1}$ and $\mathrm{B}_{2} / \mathrm{N}_{2} / \mathrm{C}_{2}$ (green and yellow curves). Raman spectra (Fig. S3 in Supplementary Information) reveal that the thickness of the BN-doped graphene is one to two layers. The enhanced intensity of STXM-XANES is associated with the reduction of the number of scattering pathways into single layer from multiple layers. This finding is expected because a thicker graphene sample is 
more likely to exhibit interactions between layers (such as a $\pi-\pi$ interaction between graphene layers) [48]. Therefore, the green and yellow regions in Fig. 3 are expected to correspond to the $\mathrm{BN}$-doped bilayer graphene, and the red regions are likely to be an approximately single layer of BN-doped graphene. According to the results in Fig. 3, the general line-shapes of the $\mathrm{B}, \mathrm{N}$ and $\mathrm{C} K$-edge STXM-XANES spectra are similar among all of the selected regions (green, yellow and red) of interest. The $\mathrm{B}_{1}-\mathrm{B}_{3}, \mathrm{~N}_{1}-\mathrm{N}_{3}$ and $\mathrm{C}_{1}-\mathrm{C}_{3}$ spectra of the $30 \% \mathrm{BN}$-doped graphene clearly demonstrates homogeneity of BN dopants therein. Importantly, Fig. 3 also reveals that the general line-shapes of the C, N and B $K$-edge STXM-XANES spectra of the $30 \% \mathrm{BN}$-doped graphene are close to those of the C, N and B $K$-edge XANES spectra of the $56 \%$ BN-doped graphene (Figs. 1, 2 and Fig. S2 in the Supplementary Information), clearly indicating the formation of $h$-BN-like domains when the $\mathrm{BN}$ concentration is relatively high. Previous TEM data [15], Raman spectroscopy and B, N and C 1 s XPS all support this scenario of the development of $h$-BN-like domains at high concentrations of BN, as shown in Figs. S2-S4 in Supplementary Information. The nonlinear behavior of bandgap opening of the $\mathrm{BN}$-doped graphene with various $\mathrm{BN}$ concentrations is also consistent with the VB-PES measurements, as discussed below.

Figure 4 presents the VB-PES spectra of graphene with various concentrations of BN 
dopants and pristine graphene, obtained using the incident photon energy of $150 \mathrm{eV}$. The near- $\pi$ region, as indicated by the downward arrow, is magnified in the inset. The two main features (labeled I and II) in the 0-5 and 6-9 eV regions, respectively, of these VB-PES spectra arise predominantly from $\mathrm{C}-\mathrm{C}$ (or $\mathrm{C}-\mathrm{N} / \mathrm{C}-\mathrm{B}$ ), $\mathrm{N}-\mathrm{B} \pi$ - and $\sigma$-bond states. However, the feature close to the $\pi$ region between 0 and $2 \mathrm{eV}$, which is magnified in the inset, is primarily associated with the C-C $\pi$ bonds $[49,50]$. This feature clearly reveals a downward shift in $E_{\mathrm{VBM}}$ or $E_{\mathrm{f}}$ in the $2 \%, 6 \%$ and $25 \%$ BN-doped graphene. The 6\% BN-doped graphene exhibits the largest downward shift $(\sim 0.4 \mathrm{eV})$ relative to $56 \% \mathrm{BN}$-doped and pristine graphene. A similar shift is observed in main feature I, which moved toward a higher binding energy, as presented in Fig. 4. A shift in the bonding states in the spectra may be caused by a change in $E_{\mathrm{VBM}}$ or $E_{\mathrm{f}}$ upon the introduction of $p$ - or $n$-type dopants, though the valence states near $E_{\mathrm{f}}$ remain constant $[13,21]$. In the present case, $\mathrm{BN}$ is isoelectronic with $\mathrm{C}$ in a similar structure, and the concentrations of $\mathrm{B}$ and $\mathrm{N}$ atoms in the graphene are almost the same, based on the B and N $1 s$ XPS (Fig. S4 and Tab. S1 in Supplementary Information). $\mathrm{B}$ and $\mathrm{N}$ doping yield $p$ - and $n$-like behaviors, respectively, and have compensating effects on the shift in $E_{\mathrm{VBM}}$ or $E_{\mathrm{f}}$, thus, their combined effects on the VB-PES spectra can be ignored. The downward shift of main feature I from the $2 \%$, $6 \%$ and $25 \%$ BN-doped graphenes, as presented in Fig. 4, reveals that electrons shift 
to higher binding energies. This result indicates that the electronic structures of the $2 \%, 6 \%$ and $25 \% \mathrm{BN}$-doped graphenes below $E_{\mathrm{VBM}}$ or $E_{\mathrm{f}}$ are modified relative to those of the $56 \% \mathrm{BN}$-doped and pristine graphene. The largest downward shift in the features of the $6 \% \mathrm{BN}$-doped graphene at/near $E_{\mathrm{VBM}}$ or $E_{\mathrm{f}}$ agrees closely with the largest bandgap obtained from the $\mathrm{C} K_{\alpha}$ XES and $K$-edge XANES spectra. The downward shift of the features in the VB-PES spectra of the $2 \%, 6 \%$ and $25 \%$ $\mathrm{BN}$-doped graphene shows $\mathrm{BN}$-doping-induced changes in the electronic states and bonding structures/phase separation in the graphene. The general line shape of main feature I and $E_{\mathrm{VBM}}$ or $E_{\mathrm{f}}$ in the VB-PES spectrum of the $56 \% \mathrm{BN}$-doped graphene displayed in Fig. 4 resembles that of pristine graphene. This result clearly suggests that the electronic structures of the $56 \% \mathrm{BN}$-doped graphene at/near $E_{\mathrm{VBM}}$ or $E_{\mathrm{f}}$ are similar to those of pristine graphene. Figure 4 presents the nonlinear shift in the rising features in the VB-PES spectra and indicates that the electronic states or bonding structures/phase separation varies with the concentration of BN dopants in graphene. This finding clearly supports the nonlinear opening of the bandgap, as presented in Fig. 1.

Theoretically, an energetically stable $\mathrm{BN}$ quantum dot (QD) domain with a hexagonally symmetric pattern has been predicted at low doping concentrations [13]. 
Various shapes of BN QDs and strip domain patterns with boundaries similar to those of graphene nanoribbons (GNR) have also been studied extensively [12,51]. To elucidate the electronic structures associated with the nonlinear opening of the bandgap of the BN-doped graphene, this work considers two possible models of BN domain geometries: (1) BN QDs with a hexagonal symmetry and various sizes embedded in a $12 \times 12$ pristine graphene hexagonal supercell and (2) an orthorhombic superlattice of up to 80 atoms with phase-separated GNR-like edges, as displayed in Fig. 5. In addition, to account for both structural stability and simple bandgap convergence [51], only the zigzag-edged structure in the BN QD is considered, whereas both zigzag and armchair edge structures are considered in the phase-separated models. The Theoretical Method above presents the theoretical details on which this work is based.

Due to the wide bandgap character of lateral $h$-BN, bandgap opening in gapless graphene is expected to occur upon doping with $\mathrm{BN}$. Replacing pairs of $\mathrm{C}$ atoms with $\mathrm{B}$ and $\mathrm{N}$ dopants yields $\mathrm{B}-\mathrm{N}$ and $\mathrm{C}-\mathrm{C}$ bonds, which have lower energies than $\mathrm{B}-\mathrm{C}$ and $\mathrm{N}-\mathrm{C}$ bonds and cause the dopant structures to be stabilized with as few C-BN interfaces as possible [13]. First, the DFT-calculated bandgaps of three BN domains [hexagonal-cluster $\mathrm{QD}\left(\mathrm{QD}_{\mathrm{Hex}}\right)$ and phase-separated nanoribbon with zigzag-edged (Zigzag) and armchair-edged (Armchair) configurations] were compared over a wide 
range of $(\mathrm{BN})_{\mathrm{x}} \mathrm{C}_{\mathrm{y}}$ doping concentrations. As presented in Fig. 6(a), the calculations predict that bandgap of graphene is gradually opened by alloying graphene with a low concentration of $\mathrm{BN} \mathrm{QD}_{\text {Hex }}$. Comparing the band structure of lower and higher doping concentrations (Figs. S5 \& S6 in Supplementary Information) reveals that a low BN dopant concentration causes a perturbation and eliminates the gapless characteristic of graphene. However, at moderate BN doping concentrations $(\sim 28 \%)$, the bandgap decreases as the dopant concentration increases. This anomalous behavior arises from the fact that the interconnection of two-dimensional (2D) graphene domains is gradually inhibited by the growth of $\mathrm{BN}$ QD islands with increasing doping concentration. These dopant-induced quantum confinement effects localized the linear dispersive $\pi$ and $\pi^{*}$ orbitals of $\mathrm{C}$ atoms at higher doping concentrations. The orbital localization reveals that $\mathrm{C}$ atoms act as substitute dopants in place of the majority $\mathrm{BN}$, thereby introducing a shallow acceptor-like defect state above the $E_{\mathrm{f}}$. The BN character dominates the calculated bandgap of $(\mathrm{BN})_{\mathrm{x}} \mathrm{C}_{y}$ because the $\mathrm{BN}$ clusters expand and yield a bandgap of $4.53 \mathrm{eV}$ at $100 \%$ doping, which is the underestimated bandgap in the DFT framework, due to the failure to account for many-body screening effects in a sophisticated manner [52-54]. Notably, the discrepancies between the experimental results and QD calculations are attributable to the many-body effects, which are not considered in the DFT studies. Ab initio 
quasiparticle GW+BSE calculations $[55,56]$ can account for the many-body effects (such as excitonic effects [28]) of these novel BNC nano-systems, although they are beyond the scope of the present work. The bandgap opening in phase-separated strips differs from that in the doping in $\mathrm{D}_{\text {Hex }}$. Figure 6(a) also shows that the bandgap of the armchair-edged nanoribbon strip increases as the concentration of the dopant increases. However, the metal-like zero-gap behavior of the zigzag-edged nanoribbon strip remains as the $\mathrm{BN}$ concentration reaches approximately $70 \%$. This result is associated mainly with the high electrical and thermal conductivity of the effective zigzag GNR of this $(\mathrm{BN})_{x} \mathrm{C}_{y}$ system. In particular, the edge state of the phase-separated strip dopant, as presented in Fig. S5 in Supplementary Information, dominates the electronic properties close to $E_{\mathrm{f}}$ and is similar to the edge state of zigzag GNR.

Following the proposal of two bandgap behaviors in $(\mathrm{BN})_{x} \mathrm{C}_{y}$, based on the DFT calculations herein, a more detailed analysis of stability of corresponding dopant geometries is required. Based on the definition in the Theoretical Method above, the difference in the formation energy per atom between $\mathrm{QD}_{H e x}$ and the zigzag-edged/armchair-edged phase-separated nanoribbon dopant is calculated. As indicated in Fig. 6(b), the calculations reveal that $\mathrm{QD}_{H e x}$ dopant is more stable than 
both the zigzag- and armchair-edged nanoribbon-type dopants at doping concentrations less than $10 \%$. Increasing the dopant concentration from $10 \%$ to $30 \%$ makes the zigzag-edged nanoribbon-type dopant energetically more stable (23 meV/atom) than $\mathrm{QD}_{H e x}$ and armchair-edged nanoribbon-type dopant. These results are also consistent with the early theoretical work [13]. As predicted, the stable dopant structures minimized the size of the interfaces between graphene and BN domains. However, the calculations herein are zero-temperature calculations, and the small difference of the corresponding formation energies per atom $\left(\Delta E_{f}\right)$ around room temperature $(26 \mathrm{meV})$ reveals that $\mathrm{QD}_{\text {Hex }}$ dopant is stabilized at moderate dopant concentrations by including thermal energies (enthalpy and entropy) in a calculation. Intermediate states are not ruled out when practical structural imperfections may enable the dopant systems to overcome activation barriers to various intermediated states. Based on the calculated behavior of bandgap, BN dopant is proposed to be present in the form of $\mathrm{QD}_{H e x}$ at low dopant concentration and then to form phase-separated zigzag-edged $\mathrm{BN}$ domains when the dopant concentration is increased, as displayed in Fig. 5. This atomic configuration is likely to maintain the interconnections within 2D graphene domains, even under high dopant concentrations, and makes BN-co-doped graphene a special platform for multifunctional bandgap engineering.5. Conclusion 
In summary, the experimental analyses and calculations from first principles reveal the nonlinear behavior of bandgap opening in $\mathrm{BN}$-doped graphene. The nonlinear behavior is a result of a variation in the interconnection of $2 \mathrm{D}$ graphene domains, which is gradually inhibited as BN QD islands grow due to an increasing doping concentration. Dopant-induced quantum confinement effects localize the linear dispersive $\pi$ and $\pi^{*}$ orbitals of $\mathrm{C}$ atoms, thus forming phase-separated domains of $\mathrm{BN}$-like and graphene at the high doping concentrations at which the $\mathrm{BN}$-doped graphene exhibit metal-like zero-gap behavior. This work paves a way for bandgap engineering in $\mathrm{BN}$-doped graphene, which is important in the development of graphene-based nanotechnology.

\section{Acknowledgement:}

The authors (H.C.H. and W.F.P.) would like to thank the Ministry of Science and Technology (MoST) of Taiwan for financially supporting this research under Contract No. MoST 104-2112-M-032-002-MY3 and NSC 102-2632-M-032-001-MY3. H.C.H. also acknowledges the support of NCTS of Taiwan. The Advanced Light Source is supported by the Director, Office of Science, Office of Basic Energy Sciences, of the U.S. Department of Energy under Contract No. DE-AC02-05CH11231. The Canadian

Light Source was supported by the Natural Sciences and Engineering Research 
Council of Canada, the National Research Council Canada, the Canadian Institutes of

Health Research, the Province of Saskatchewan, Western Economic Diversification Canada, and the University of Saskatchewan.

Appendix A. Supplementary data

Supplementary data related to this article can be found at http://...

\section{References:}

1 A.K. Geim, K.S. Novoselov, The rise of graphene, Nature Mater. 6 (2007) 183-191.

2 K. Kim, J.Y. Choi, T. Kim, S.H. Cho, H.J. Chung, A role for graphene in silicon-based semiconductor devices, Nature 479 (2011) 338-344.

3 M.Y. Han, B. Özyilmaz, Y. Zhang, P. Kim, Energy band-gap engineering of graphene nanoribbons, Phys. Rev. Lett. 98 (2007) 206805.

4 T. Ohta, A. Bostwick, T. Seyller, K. Horn, E. Rotenberg, Controlling the electronic structure of bilayer graphene, Science 313 (2006) 951-954.

5 D.C. Elias, R.R. Nair, T.M.G. Mohiuddin, S.V. Morozov, P. Blake, M.P. Halsall, etal., Control of graphene's properties by reversible hydrogenation: Evidence for graphane, Science 323 (2009) 610-613.

6 J.T. Robinson, J.S. Burgess, C.E. Junkermeier, S.C. Badescu, T.L. Reinecke, F.K. Perkins, et al., Properties of Fluorinated Graphene Films, Nano Lett. 10 (2010) 3001-3005. 
7 R.R. Nair, W. Ren, R. Jalil, I. Riaz, V.G. Kravets, L. Britnell, et al., Fluorographene: A Two-Dimensional Counterpart of Teflon, Small 6 (2010) 2877-2884.

8 R. Zbořil, F. Karlický, A.B. Bourlinos, T.A. Steriotis, A.K. Stubos, V. Georgakilas, et al., Graphene Fluoride: A Stable Stoichiometric Graphene Derivative and its Chemical Conversion to Graphene, Small 6 (2010) 2885-2891.

9 D. Usachov, O. Vilkov, A. Grüneis, D. Haberer, A. Fedorov, V.K. Adamchuk, et al., Nitrogen-doped graphene: Efficient growth, structure and electronic properties, Nano Lett. 11 (2011) 5401-5407.

10 K. Watanabe, T. Taniguchi, H. Kanda, Direct-bandgap properties and evidence for ultraviolet lasing of hexagonal boron nitride single crystal, Nature Mater. 3 (2004) 404-409.

11 M. Kan, J. Zhou, Q. Wang, Q. Sun, P. Jena, Tuning the band gap and magnetic properties of BN sheets impregnated with graphene flakes, Phys. Rev. B 84 (2011) 205412.

12 P.P. Shinde, V. Kumar, Direct band gap opening in graphene by BN doping: $A b$ initio calculations, Phys. Rev. B 84 (2011) 125401.

13 X. Fan, Z. Shen, A.Q. Liu, J.L. Kuo, Bandgap opening of graphene by doping small boron nitride domains, Nanoscale 4 (2012) 2157-2165.

14 L. Ci, L. Song, C. Jin, D. Jariwala, D. Wu, Y. Li, et al., Atomic layers of hybridized boron nitride and graphene domains, Nature Mater. 9 (2010) 430-435. 
15 C.K. Chang, S. Kataria, C.C. Kuo, A. Ganguly, B.Y. Wang, J.Y. Hwang, et al., Bandgap engineering of chemical vapor deposited graphene by in-situ BN doping, ACS Nano 7 (2013) 1333-1341.

16 G.C. Loh, R. Pandey, A graphene-boron nitride lateral heterostructure, J. Mater. Chem. C 3 (2015) 5918.

17 P. Nath, S. Chowdhury, D. Sanyal, D. Jana, Ab-initio calculation of electronic and optical properties of nitrogen and boron doped graphene nanosheet, Carbon $\mathbf{7 3}$ (2014) 275-282.

18 R. Nascimento, J. da R. Martins, R.J.C. Batista, H. Chacham, Band Gaps of BN-Doped Graphene Fluctuations, Trends, and Bounds, J. Phys. Chem. C 119 (2015) 5055-5061.

19 L. Ferrighi, M.I. Trioni, C.D. Valentin, Boron-Doped, Nitrogen-Doped and Codoped Graphene on Cu(111): A DFT+VdW Study, J. Phys. Chem. C 119 (2015) 6056-6064.

20 P. Rani, V.K. Jindal, Designing band gap of graphene by B and N dopant, RSC Advances 3 (2013) 802.

21 V.L. Solozhenko, A.G. Lazarenko, J.P. Petitet, A.V. Kanaev, Bandgap energy of graphite-like hexagonal boron nitride, J. Phys. Chem. Solids 62 (2001) $1331-1334$. 
22 J.B. MacNaughton, A. Moewes, R.G. Wilks, X.T. Zhou, T.K. Sham, T. Taniguchi, et al., Electronic structure of boron nitride single crystals and films, Phys. Rev. B 72 (2005) 195113.

23 L. Song, L. Ci, H. Lu, P.B. Sorokin, C. Jin, J. Ni, et al., Large scale growth and characterization of atomic hexagonal boron nitride layers, Nano Lett. 10 (2010) 3209-3215.

24 K.K. Kim, A. Hsu, X. Jia, S.M. Kim, Y. Shi, M. Hofmann, et al., Synthesis of monolayer hexagonal boron nitride on $\mathrm{Cu}$ foil using chemical vapor deposition, Nano Lett. 12 (2012) 161-166.

25 Y. Gao, W. Ren, T. Ma, Z. Liu, Y. Zhang, W.B. Liu, et al., Repeated and controlled growth of monolayer, bilayer and few-layer hexagonal boron nitride on Pt foils, ACS Nano. 7 (2013) 5199-5206.

26 I. Gierz, C. Riedl, U. Starke, C.R. Ast, K. Kern, Atomic hole doping of graphene, Nano Lett. 8 (2008) 4603-4607.

27 Z.M. Liu, Y. Zhu, Z.Q. Yang, Half metallicity and electronic structures in armchair BCN-hybrid nanoribbons, J. Chem. Phys. 134 (2011) 074708.

28 M. Bernardi, M. Palummo, J.C. Grossman, Optoelectronic properties in monolayers of hybridized graphene and hexagonal boron nitride, Phys. Rev. Lett. 108 (2012) 226805. 
29 Z.H. Huang, V.H. Crespi, J.R. Chelikowsky, Electronic properties of mixed-phase graphene/h-BN sheets using real-space pseudopotentials, Phys. Rev. B 88 (2013) 235425 .

30 Z. Wang, J. Wang, T.K. Sham, S. Yang, Origin of luminescence from $\mathrm{ZnO/CdS}$ core/shell nanowire arrays, Nanoscale 6 (2014) 9783-9790.

31 S.B. Singh, W.F. Wang, Y.C. Shao, H.Y. Lai, S.H. Hsieh, M.V. Limaye, et al., Observation of the origin of $\mathrm{d}^{0}$ magnetism in $\mathrm{ZnO}$ nanostructures using X-ray-based microscopic and spectroscopic techniques, Nanoscale 6 (2014) 9166-9176.

32 G. Kresse, D. Joubert, From ultrasoft pseudopotentials to the projector augmented-wave method, Phys. Rev. B 59, 1758 (1999).

33 G. Kresse, J. Furthmüller, Efficient iterative schemes for $a b$ initio total-energy calculations using a plane-wave basis set, Phys. Rev. B 54 (1996) 11169.

34 P.E. Blöchl, Projector augmented-wave method, Phys. Rev. B 50 (1994) 17953.

35 H.J. Monkhorst, J.D. Pack, Special points for Brillouin-zone integrations, Phys. Rev. B 13 (1976) 5188. 
36 C.W. Pao, S.C. Ray, H.M. Tsai, Y.S. Chen, H.C. Chen, I.N. Lin, et al., Change of structural behaviors of organo-silane exposed graphene nanoflakes, J. Phys. Chem. C 114 (2010) 8161-8166.

37 J.W. Chiou, S.C. Ray, S.I. Peng, C.H. Chuang, B.Y. Wang, H.M. Tsai, et al., Nitrogen-functionalized graphene nanoflakes (GNFs: N): Tunable photoluminescence and electronic structures, J. Phys. Chem. C 116 (2012) 16251-16258.

38 C.H. Chuang, Y.F. Wang, Y.C. Shao, Y.C. Yeh, D.Y. Wang, C.W. Chen, et al., The effect of thermal reduction on the photoluminescence and electronic structures of graphene oxides, Sci. Reps. 4 (2014) 4525.

39 S. Rajasekaran, S. Kaya, T. Anniyev, H. Ogasawara, A. Nilsson, Probing substrate effects in the carbon-projected band structure of graphene on Pt (111) through resonant inelastic x-ray scattering, Phys. Rev. B 85 (2012) 045419.

40 D. Pacilé, M. Papagno, A.F. Rodríguez, M. Grioni, L. Papagno, C.Ö Girit, et al., Near-edge x-ray absorption fine-structure investigation of graphene, Phys. Rev. Lett. 101 (2008) 066806.

$41 \mathrm{~K}$. Yuge, Phase stability of boron carbon nitride in a heterographene structure: A first-principles study, Phys. Rev. B 79 (2009) 144109. 
42 J.G. Zhou, J. Wang, C.L. Sun, J.M. Maley, R. Sammynaiken, T.K. Sham, et al., Nano-scale chemical imaging of a single sheet of reduced graphene oxide, J. Mater. Chem. 21 (2011) 14622-14630.

43 H.K. Jeong, H.J. Noh, J.Y. Kim, L. Colakerol, P.A. Glans, M.H. Jin, et al., Comment on "Near-edge $\mathrm{x}$-ray absorption fine structure investigation of graphene”, Phys. Rev. Lett. 102 (2009) 099701.

44 C.W. Pao, C.T. Wu, H.M. Tsai, Y.S. Liu, C.L. Chang, W.F. Pong, et al., Photoconduction and the electronic structure of silica nanowires embedded with gold nanoparticles, Phys. Rev. B 84 (2011) 165412.

45 M. Lerotic, C. Jacobsen, T. Schäfer, S. Vogt, Cluster analysis of soft X-ray spectromicroscopy data, Ultramicroscopy 100 (2004) 35-37.

46 M. Lerotic, C. Jacobsen, J.B. Gillow, A.J. Francis, S. Wirick, S. Vogt, et al., Cluster analysis in soft X-ray spectromicroscopy: Finding the patterns in complex specimens, J. Elect. Spectro. \& Related Phenom. 144 (2005) 1137-1143.

47 http://unicorn,mcmaster.ca/aXis2000.html

48 J.G. Zhou, J. Wang, C.L. Sun, J.M. Maley, R. Sammynaiken, T.K. Sham, et al., Nano-scale chemical imaging of a single sheet of reduced graphene oxide, J. Mater. Chem. 21 (2011) 14622-14630.

49 S. Souto, M. Pickholz, M.C. dos Santos, F. Alvarez, Electronic structure of nitrogen-carbon alloys $\left(a-\mathrm{CN}_{\mathrm{x}}\right)$ determined by photoelectron spectroscopy, Phys. Rev. B 57 (1998) 2536. 
50 S.C. Ray, H.M. Tsai, C.W. Bao, J.W. Chiou, J.C. Jan, K.P. Krishna Kumar, et al., Electronic and bonding structures of B-C-N thin films investigated by $\mathrm{x}$-ray absorption and photoemission spectroscopy, J. Appl. Phys. 96 (2004) 208.

51 R. Zhao, J. Wang, M. Yang, Z. Liu, Z. Liu, BN-embedded graphene with a ubiquitous gap opening, J. Phys .Chem. C 116 (2012) 21098-21103.

52 L. Hedin, New method for calculating the one-particle Green's function with application to the electron-gas problem, Phys. Rev. A 139 (1965) 796.

53 M. S. Hybertsen, S.G. Louie, Electron correlation in semiconductors and insulators: Band gaps and quasiparticle energies, Phys. Rev. B 34 (1986) 5390.

54 W.G. Aulbur, L. Jonsson, J.W. Wilkins, Quasiparticle calculations in solids. Solid State Physics: Advances in Research and Applications 54 (2000) 1.

55 M. Rohlfing, S.G. Louie, Electron-hole excitations and optical spectra from first principles, Phys. Rev. B 62 (2000) 4927.

56 H.C. Hsueh, G.Y. Guo, S.G. Louie, Excitonic effects in the optical properties of a SiC sheet and nanotubes, Phys. Rev. B 84 (2011) 085404. 


\section{Figure Captions}

Fig. 1 (a) Normalized C $K_{\alpha}$ XES and $K$-edge XANES spectra of BN-doped graphene with various BN concentrations, pristine graphene and HOPG. (b) Magnified $\pi-\pi^{*}$ regions of spectra. (c) Scheme of bandgap obtained from the energy between $E_{\mathrm{VBM}}$ (valence band maximum) and $E_{\mathrm{CBM}}$ (conduction band minimum) (preliminary result has been published in Ref. 15).

Fig. $2 \mathrm{~N} K_{\alpha}$ XES and $K$-edge XANES spectra of variously BN-doped graphene. Maximum intensities of features in XES and XANES spectra of $56 \%$ BN-doped graphene and $h$-BN are normalized to be equal.

Fig. 3 STXM mappings of B, N and C in 30\% BN-doped graphene. STXM images $\left(\mathrm{B}_{0}, \mathrm{~N}_{0}\right.$ ad $\mathrm{C}_{0}$ ) (left panels) of blue regions are obtained from a background area of samples. Green, yellow and red regions $\left(\mathrm{B}_{1}-\mathrm{B}_{3}, \mathrm{~N}_{1}-\mathrm{N}_{3}\right.$ and $\left.\mathrm{C}_{1}-\mathrm{C}_{3}\right)$ in the middle panels are associated with various thicknesses of graphene. Right panels display corresponding STXM-XANES spectra.

Fig. 4 VB-PES spectra of BN-doped graphene with various $\mathrm{BN}$ concentrations and pristine graphene obtained using the incident photon energy of $150 \mathrm{eV}$. Inset magnifies the near- $\pi$ region $(0-2 \mathrm{eV})$ for comparison. 
Fig. 5 Supercells employed in calculations of electronic structures of (a) $(\mathrm{BN})_{3} \mathrm{C}_{282}$ (b) $(\mathrm{BN}){ }_{12} \mathrm{C}_{264}$ in $\mathrm{BN} \quad \mathrm{QD}_{(\mathrm{Hex})}$, and (c) zigzag-edged phase-separated $(\mathrm{BN})_{4} \mathrm{C}_{16}$, respectively. The corresponding periodic unit cells are presented in red-dashed boxes. Unit cell in (c) is enlarged, and C, B, and $\mathrm{N}$ atoms are shown as small yellow, large purple, and small light blue spheres, respectively.

Fig. 6 (a) DFT-calculated band gaps of $(\mathrm{BN})_{x} \mathrm{C}_{\mathrm{y}}$ for $\mathrm{BN}$ domains in $\mathrm{QD}_{(\text {Hex })}$ (black diamonds), zigzag-edged phase-separated nanoribbon strip (red circles), and armchair-edged phase-separated nanoribbon strip (blue squares) as functions of dopant concentration. Lines that connect data are guides for the eye. Experimental data (green triangles) are presented for comparison. (b) Difference between energy of formation of $\mathrm{QD}_{(H e x)}$ and that of zigzag-edged/armchair-edged nanoribbon-type dopants as function of dopant concentration. Structural stability of both nanoribbon-type dopants, which is related to $\mathrm{QD}_{(\text {Hex })}$ dopant, can be estimated from crossovers between red/blue lines and the black line. 
figure 1
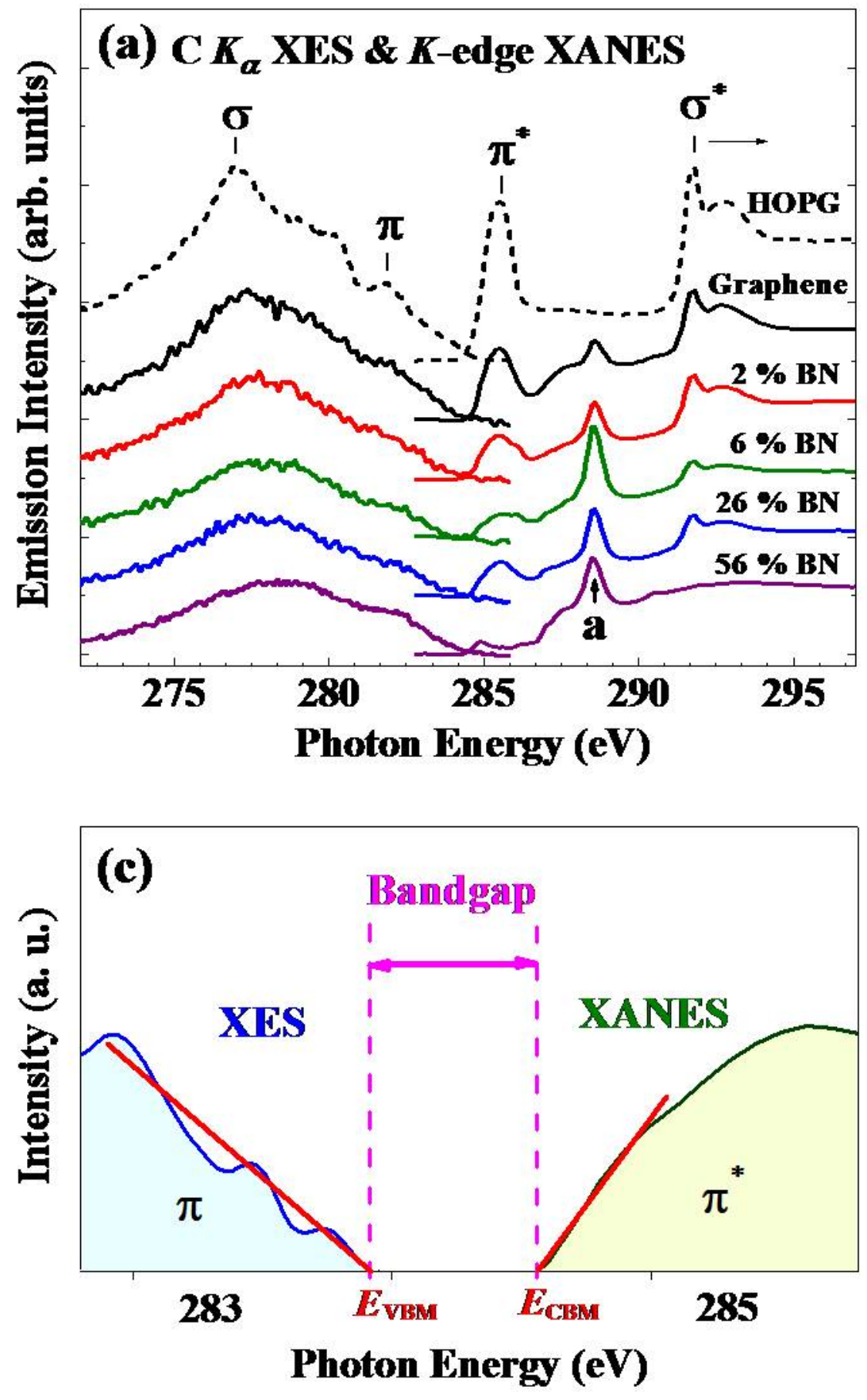

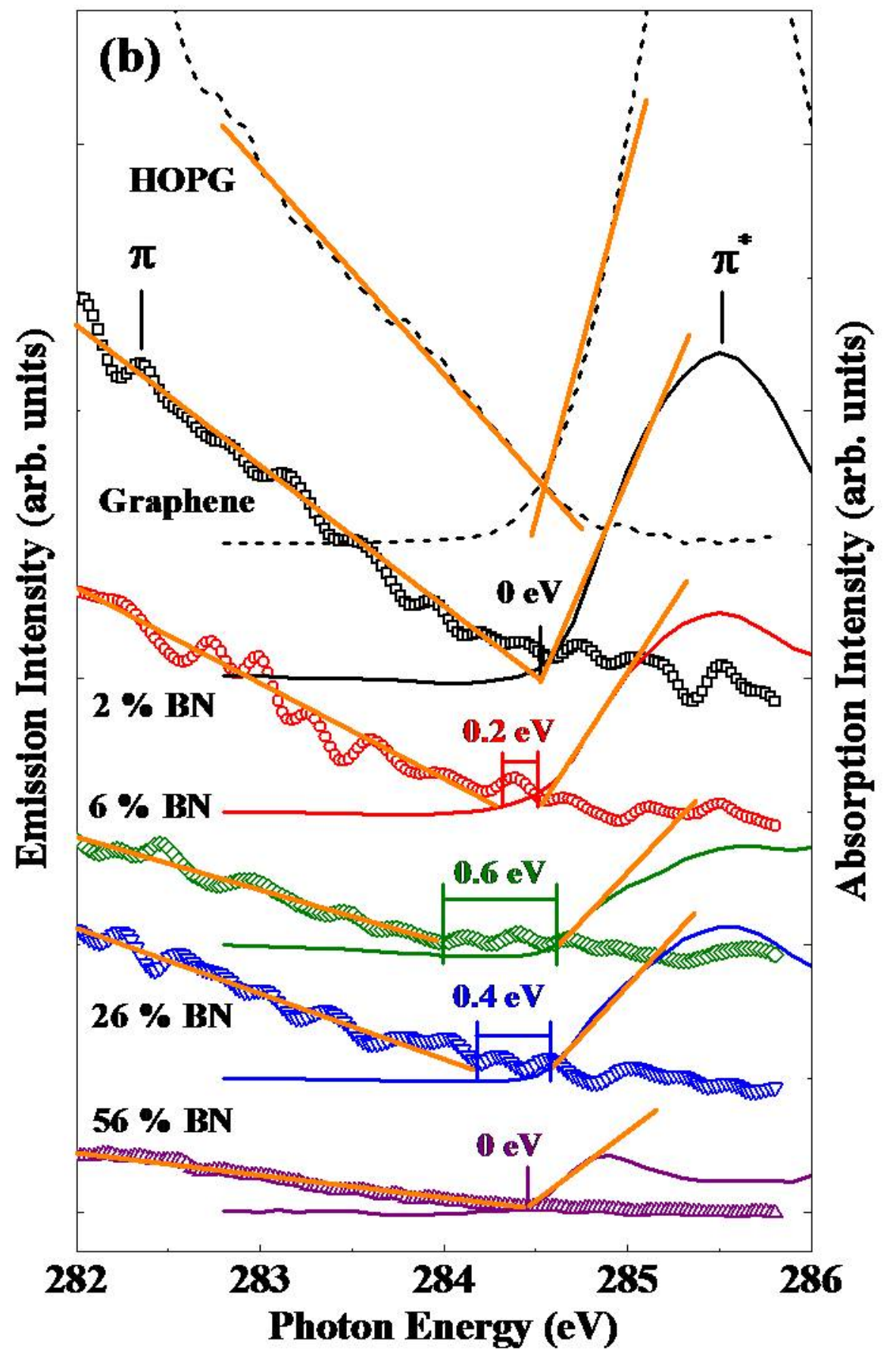


figure 2

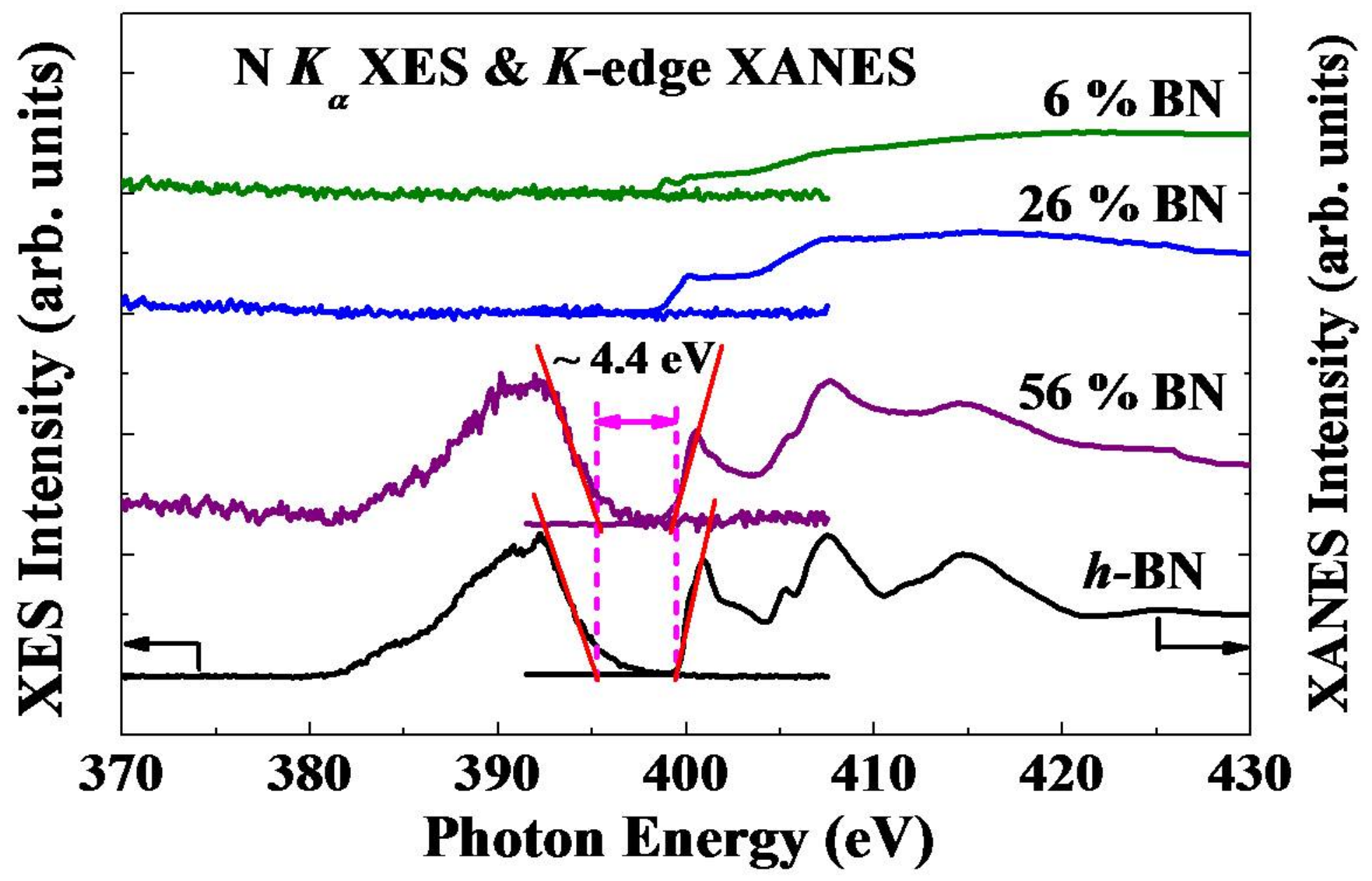


figure 3

B $K$-edge STXM

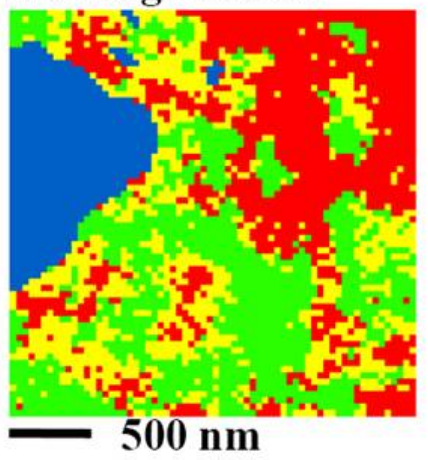

\section{N $K$-edge STXM}

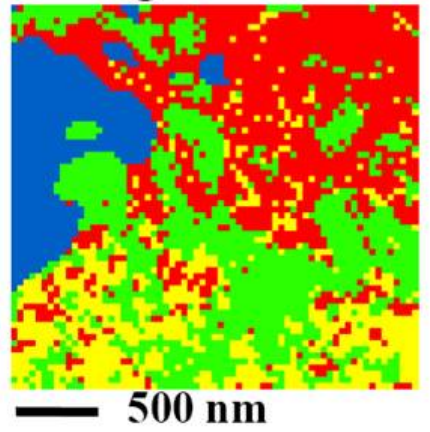

\section{K-edge STXM}

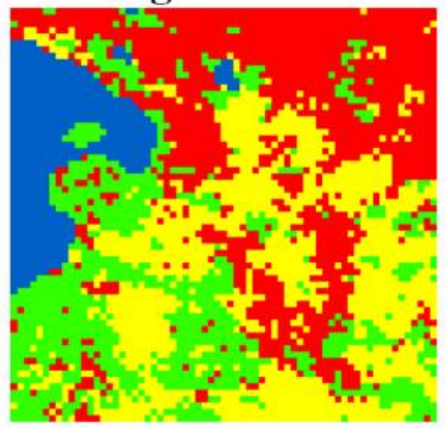

$-500 \mathrm{~nm}$
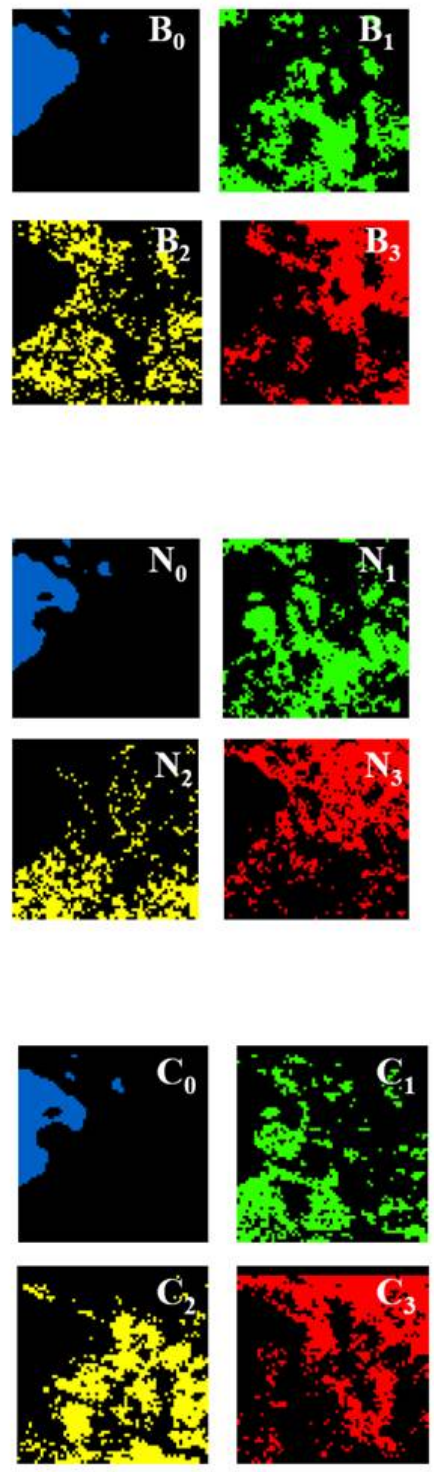
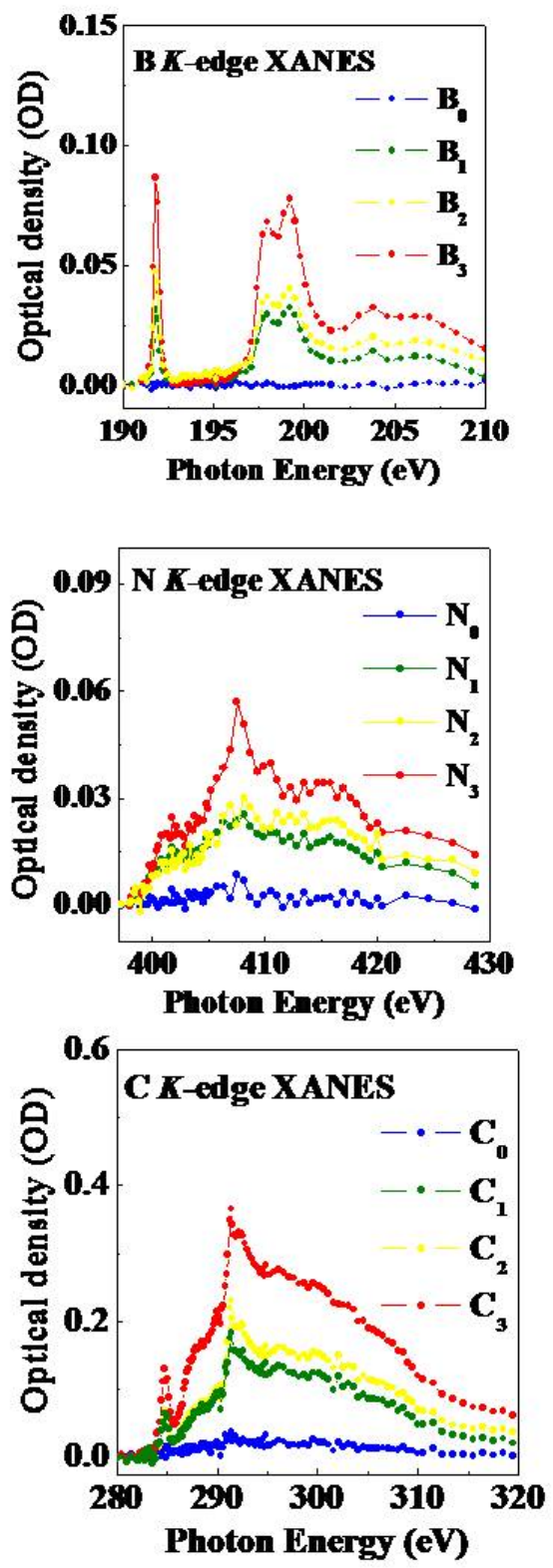
figure 4

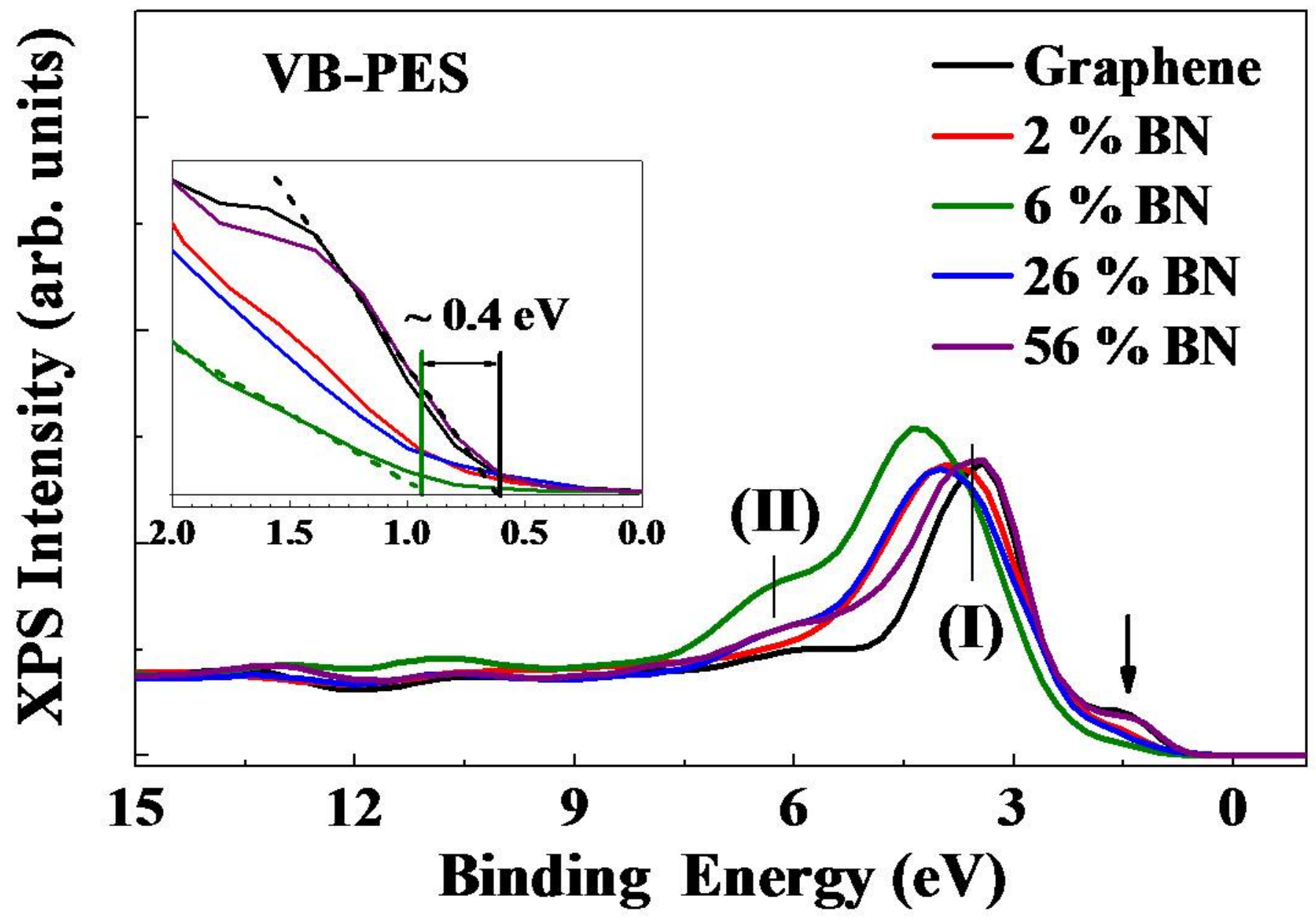


figure 5

(a)

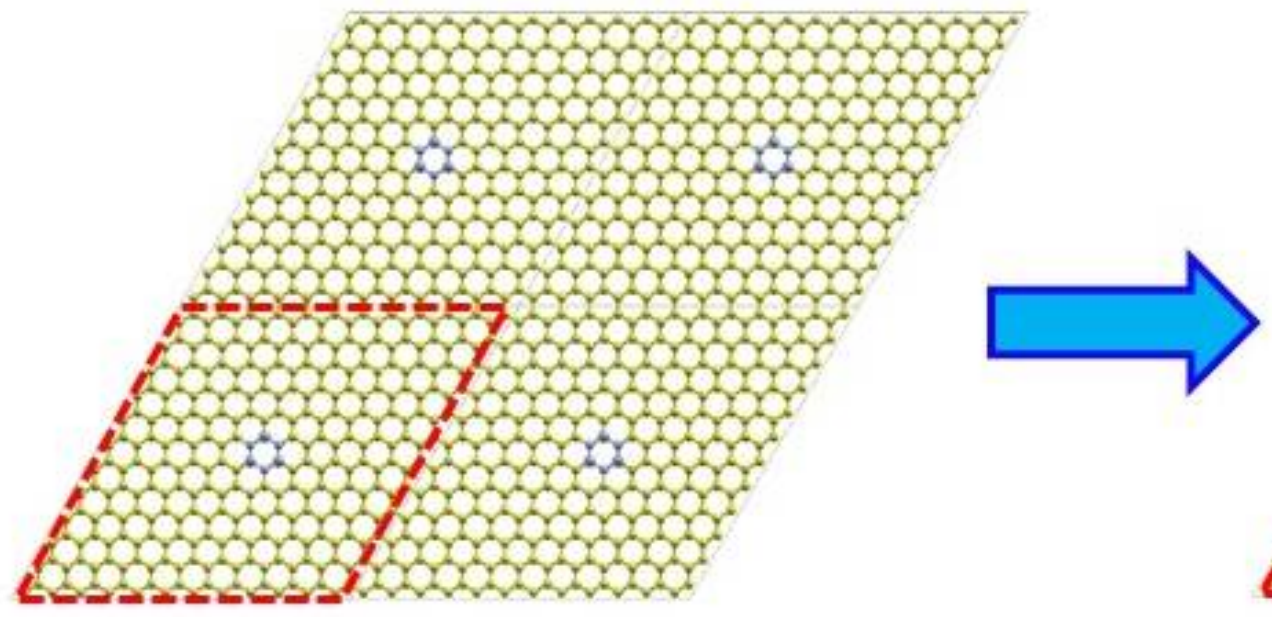

(b)

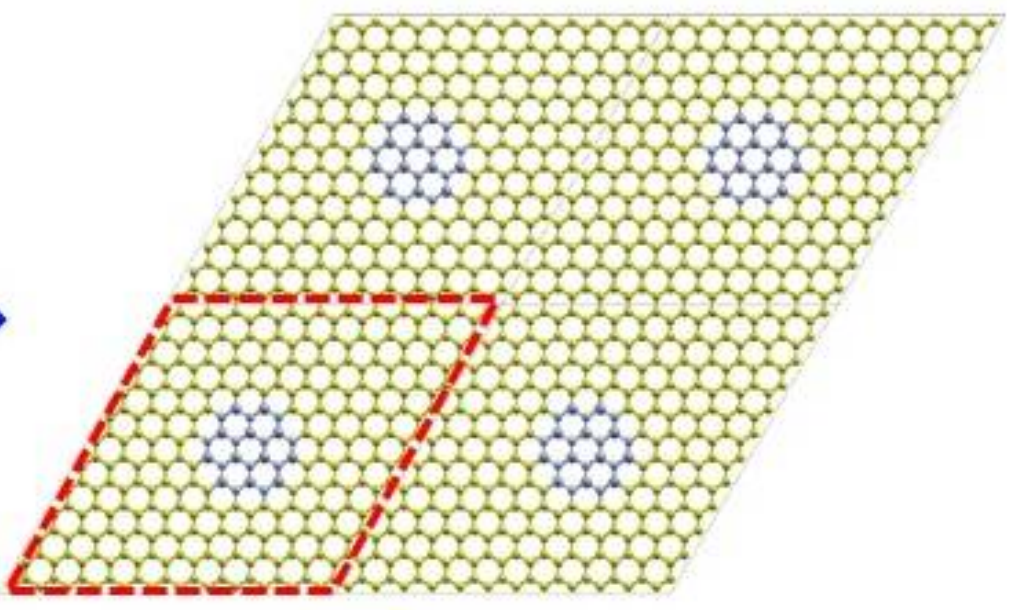

(c)

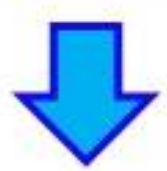

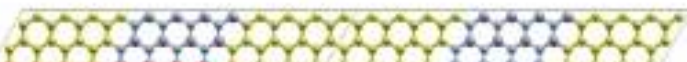

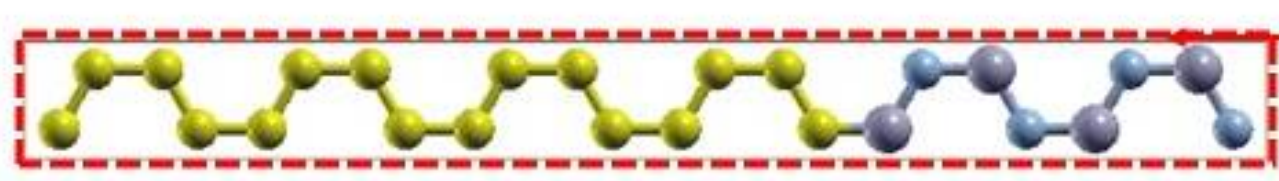

hithis

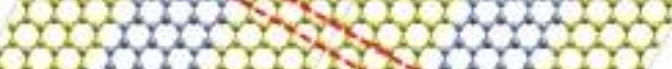

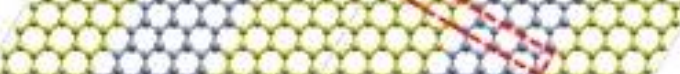

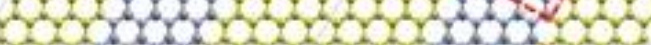
mar e

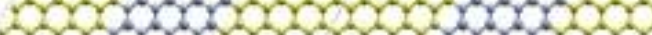

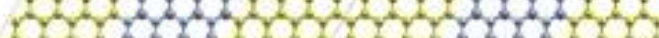

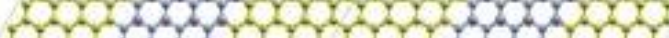

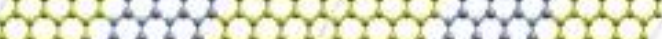

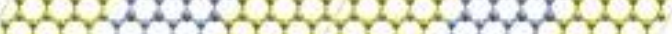

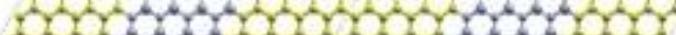

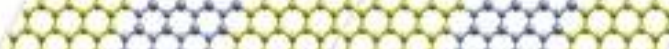


figure 6
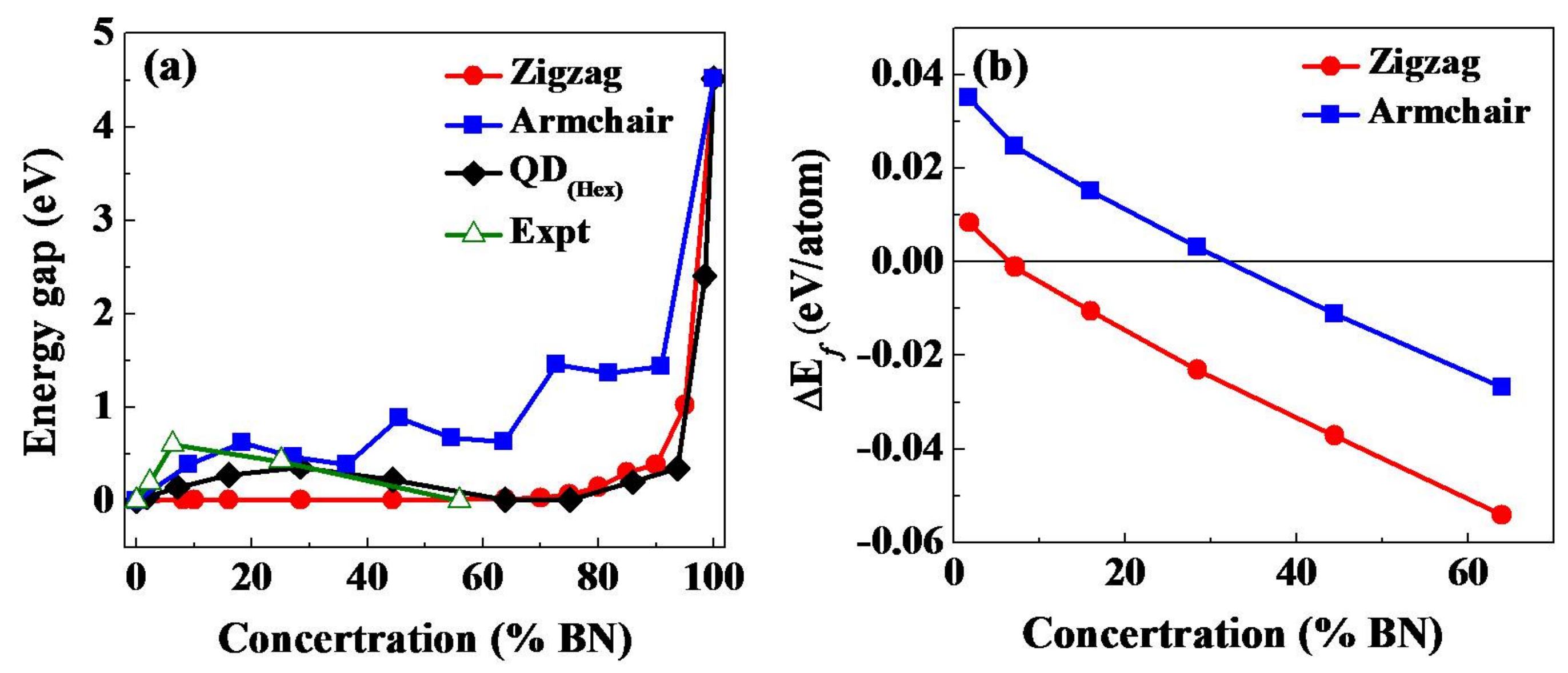